\title{
A note on periodic solutions in non-symmetric May-Leonard systems
}

\author{
Zsolt Szentkuti
}




\title{
A NOTE ON PERIODIC SOLUTIONS IN NON-SYMMETRIC MAY-LEONARD SYSTEMS
}

\author{
Zsolt SzentKuti \\ Budapest University of Technology and Economics, Institute of Mathematics \\ H-1521 Budapest, P.O.B.91., Hungary \\ szs@math. bme.hu
}

[Received August 29, 2000]

\begin{abstract}
We shall give a proof in this extension of our previous paper for the conjecture about the occurrence of periodic orbits by means of Hopf bifurcation in non-symmetric May-Leonard systems.

Mathematical Subject Classification: 92D40, 34Cxx

Keywords: Periodic orbitsd, Hopf bifurcation
\end{abstract}

\section{Introduction}

This paper is the continuation of our previous considerations in which we have studied the occurrence of periodic orbits by means of Hopf bifurcation in non-symmetric May-Leonard systems ([5], [8]). In the previous work we gave a conjecture through graphical analysis of the null-clines and we shall give a proof in the present work. In the meantime the valuable results of Chia-Wei Chi, Sze-Bi Hsu and Lih-Ing Wu ([7]) were published in which they observed the global behavior of a more general threedimensional non-symmetric system than it was shown in our paper. Their results contain our former findings about a special case of the non-symmetric May-Leonard model.

We have analyzed in the previous paper when a Hopf bifurcation occurs in the following special case of the Lotka-Volterra model ([1]):

$$
\left.\begin{array}{l}
\frac{d x_{1}}{d t}=x_{1} \cdot\left(1-x_{1}-\alpha \cdot x_{2}-\gamma \cdot x_{3}\right) \\
\frac{d x_{2}}{d t}=x_{2} \cdot\left(1-\beta \cdot x_{1}-x_{2}-\alpha \cdot x_{3}\right) \\
\frac{d x_{3}}{d t}=x_{3} \cdot\left(1-\alpha \cdot x_{1}-\beta \cdot x_{2}-x_{3}\right)
\end{array}\right\}
$$

with the conditions:

$$
0<\alpha<1, \quad \beta>1, \quad \gamma>1
$$

May and Leonard [2] first observed the symmetric case (it means that $\gamma=\alpha$ ) of the system (1.1) and they showed:

1. if $\alpha+\beta<2$, then there is an internal fixed point $P$ in the positive cone $R_{+}^{3}$ and $P$ is stable, 
2. if $\alpha+\beta \geq 2$ and also $\alpha>1$ and $\beta>1$, then the system converges to a fixed point, denoted by $R_{i}(i=1,2,3)$ lying on any of the coordinate axes. Fixed points of this type are called axial fixed points,

3. if $\alpha+\beta \geq 2$ and also $\alpha<1$ and $\beta>1$ (or $\alpha>1$ and $\beta<1$, which is a totally symmetric case), then no stable fixed point exists.

May, Leonard and Schuster, Sigmund and Wolf [2], [3] showed that in the last case the system exhibits a general class of solutions with non-periodic oscillations of bounded amplitude and increasing cycle time.

The third is the most interesting case for our recent considerations and we call the system May-Leonard system if the conditions in the third case hold. (We supposed the condition (1.2) because of this.) We will analyze the system and sketch the possible phase-portraits for this model in Section 2. We shall give a necessary and sufficient condition for the occurrence of Hopf bifurcation. We will use by this consideration the classification theory of M. L. Zeeman for three-dimensional Lotka-Volterra models [4], [6]. We will give a summary about the global behavior of the general case of nonsymmetric May-Leonard systems in Section 3.

\section{Occurrence of periodic orbits in the special case}

We will study in this section the global behavior of system (1.1) with condition (1.2) on the carrying simplex. For the sake of completeness we will observe the case $0<\gamma<1$ at the end of the section. Let us first compute the equilibrium points of the model:

1. the origin: $O=\operatorname{col}[0,0,0]$,

2. the axial fixed points: $R_{1}: \operatorname{col}[1,0,0], R_{2}: \operatorname{col}[0,1,0]$ and $R_{3}: \operatorname{col}[0,0,1]$,

3. the planar fixed points: $Q_{1}: \frac{1}{\alpha \cdot \beta-1} \operatorname{col}[0, \alpha-1, \beta-1], Q_{2}: \frac{1}{\alpha \cdot \gamma-1} \operatorname{col}[\gamma-$ $1,0, \alpha-1]$ and $Q_{3}: \frac{1}{\alpha \cdot \beta-1} \operatorname{col}[\alpha-1, \beta-1,0]$,

4. the interior fixed point:

$$
\begin{array}{r}
P: \frac{1}{\operatorname{det} A} \operatorname{col}\left[\alpha^{2}-\alpha \beta-\alpha+1+\gamma \beta-\gamma, 1-\alpha-\gamma \alpha-\beta+\alpha^{2}+\gamma \beta\right. \\
\left.1-\alpha \beta+\alpha^{2}-\alpha-\beta+\beta^{2}\right]=\frac{1}{\operatorname{det} A}(a, b, c)
\end{array}
$$

where $\operatorname{det} A=1-2 \alpha \beta-\gamma \alpha+\alpha^{3}>0$ and $a, b, c$ are the coordinates of the interior fixed point. A later computation shows that $a>0, b>0, c>0, \operatorname{det} A>0$ and because of this $P$ is lying in the positive cone.

Considering conditions (1.2) we can see that by these assumptions the planar fixed points do not lie in the positive cone. Computing the $R_{i j}$ and $Q_{i i}$ expressions $(i, j=1,2,3)$ for the classification of M. L. Zeeman ([4]) we get:

$$
\begin{array}{ccc}
R_{12}=1, & R_{21}=-1, & R_{31}=1, \\
R_{13}=-1, & R_{23}=1, & R_{32}=-1 .
\end{array}
$$



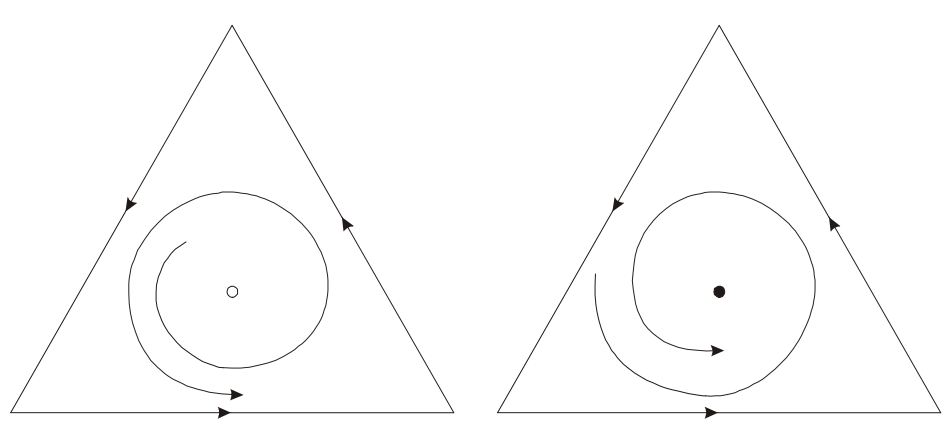

Figure 1. Possible phase

On the basis of the preceding considerations we can classify the system into a stable null-cline class and we can draw two possible phase portraits for the system on the carrying simplex (FIGURE 1) representing the global long-term behavior (it is represented for simplicity on the unit simplex which is homeomorphic with the real phase portrait).

If we want to say something about the occurrence of Hopf bifurcation, we have to compute the varying system. The varying system of system (1.1) is the following:

$$
\dot{y}=\frac{1}{\operatorname{det} A}\left[\begin{array}{ccc}
-a & -\alpha a & -\gamma a \\
-\beta b & -b & -\alpha b \\
-\alpha c & -\beta b & -c
\end{array}\right] \cdot y=\frac{1}{\operatorname{det} A}\left[\begin{array}{ccc}
-a & 0 & 0 \\
0 & -b & 0 \\
0 & 0 & -c
\end{array}\right] \cdot\left[\begin{array}{ccc}
1 & \alpha & \gamma \\
\beta & 1 & \alpha \\
\alpha & \beta & 1
\end{array}\right] \cdot y
$$

where $\operatorname{det} A>0$ and $a, b, c>0$. Computing the eigenvalues of the varying system $(2.1)$ :

$$
\begin{aligned}
& \lambda_{1}=-1 \\
& \lambda_{2,3}=\frac{1}{2}\left[(1-a-b-c) \pm \sqrt{(a+b+c-1)^{2}-4 a b c(\operatorname{det} A)}\right]
\end{aligned}
$$

By studying the (2.2) eigenvalues of the varying system we can find the following:

Theorem 1 In system (1.1) there occurs a Hopf bifurcation if and only if the expression

$$
\gamma=1-\frac{(\alpha-1)^{3}}{(\beta-1)^{2}}
$$

holds.

Proof. Let us consider how the conditions of the Andronov-Hopf theorem [1] hold. 
1. Condition $\operatorname{Re} \lambda_{1}<0$ is fulfilled because $\lambda_{1}=-1$.

2. Secondly, we need to observe the two other eigenvalues. Let us use the following notations:

$$
\begin{gathered}
\Delta=\operatorname{det} A, \quad \Delta_{1}=\operatorname{det}\left[\begin{array}{ccc}
1 & \alpha & \gamma \\
1 & 1 & \alpha \\
1 & \beta & 1
\end{array}\right], \quad \Delta_{2}=\operatorname{det}\left[\begin{array}{lll}
1 & 1 & \gamma \\
\beta & 1 & \alpha \\
\alpha & 1 & 1
\end{array}\right], \\
\Delta_{3}=\operatorname{det}\left[\begin{array}{lll}
1 & \alpha & 1 \\
\beta & 1 & 1 \\
\alpha & \beta & 1
\end{array}\right]
\end{gathered}
$$

and $A=1-\alpha>0, B=\beta-1>0, C=\gamma-1>0$. Using these notations a simple computation yields:

$$
\begin{aligned}
& \Delta=B^{2} C+2 B C+B^{2}+2 A B+A C+A^{2}(3-A)>0 \\
& \Delta_{1}=A^{2}+A B+B C>0 \\
& \Delta_{2}=A^{2}+A C+B C>0 \\
& \Delta_{3}=A^{2}+A B+B^{2}>0
\end{aligned}
$$

We can easily see with these results that the interior fixed point is lying in the positive cone:

$$
P:(a, b, c)=\left(\frac{\Delta_{1}}{\Delta}, \frac{\Delta_{2}}{\Delta}, \frac{\Delta_{3}}{\Delta}\right)>0
$$

The real part of $\lambda_{2,3}$ is $\frac{1}{2}(1-a-b-c)$ because

$$
(a+b+c-1)^{2}-4 a b c(\operatorname{det} A)<0
$$

Let us prove this inequality:

$$
\begin{aligned}
& (a+b+c-1)^{2}-4 a b c(\operatorname{det} A)=\frac{1}{\Delta^{2}}\left[\left(\Delta_{1}+\Delta_{2}+\Delta_{3}-\Delta\right)^{2}-4 \Delta_{1} \Delta_{2} \Delta_{3}\right]= \\
& \frac{1}{\Delta^{2}}\left[\left(B^{2} C-A^{3}\right)^{2}-4\left(A^{2}+A B+B C\right)\left(A^{2}+A C+B C\right)\left(A^{6}+A B+B^{2}\right)\right]= \\
& \frac{1}{\Delta^{2}}\left[B^{4} C^{0}+A^{6}-2 A^{2} B^{2} C-4\left(B^{4} C^{2}+A^{6}+G(A, B, C)\right]<0\right.
\end{aligned}
$$

where $\mathrm{G}$ is a homogeneous polynomial of A, B, $\mathrm{C}$ and $G>0$. Since the inequality holds, $\lambda_{2,3}$ has not only real parcs. Let us observe the following bifurcation parameter:

$$
\mu=1-(a+b+c)
$$

The real part of $\lambda_{2,3}$ is with this parameter:

$$
\operatorname{Re} \lambda_{2,3}=\frac{\mu}{2} \quad \text { and } \quad \frac{d \operatorname{Re} \lambda_{2,3}}{d \mu}=\frac{1}{6}>0 .
$$

All of the conditions of the Andronov-Hopf theorem are fulfilled. 


\section{Summary}

We could see previously a simple generalization of the symmetrical May-Leonard model, which neglects eonsidering symmetry. The papers mentioned in the introduction give stability results also for models more general than system (1.1). In our previous and current paper we examined which conditions should hold for periodic orbits to occur in the model by means of Hopf bifurcation. We gave a necessary and sufficient condition for Hopf bifurcation to occur in the above non-symmetric system, and our results coincided with the more general case restricted to model (1.1) with (1.2). Based sn this fact we can say that the stability loss of the equilibrium state of the system by the specified conditions is due to the presence of periodic orbits occurring by means of Hopf bifurcation.

\section{REFERENCES}

[1] Farkas, M.: Periodic Motions, Springer-Verlag, New York, 1994.

[2] May, R. M.: Nonlinear aspects of competition between three species, SIAM Journal of Applied Mathematics, 29, (1975), 243-253.

[3] Schuster, P., Sigmund, K. And Wolff, R.: On w-limits for competition between three species, SIAM Journal of Applied Mathematics, 37, (1979), 49-54.

[4] Zeeman, M. L.: Hopf bifurcations in Lotka-Volterra systems, Dynamics and Stability of Systems, 8, (1993) 189-217.

[5] Szentkuti, Zs.: Periodic solutions in May-Leonard type population dynamic models, Alkalmazott Matematikai Lapok, 19 , (1998), 23-38 (In Hungarian).

[6] Van Den Driesche, P., Zeeman, M. L.: Three-dimensional competitive Lotka-Volterra systems with non-periodic orbits, SIAM Journal of Applied Mathematics, 58, (1998), $227-234$.

[7] Chia-Wei Chi, Sze-Bi Hsu, Lin-Ing Wu: On the asymmetric May-Leonard model of three competing species, SIAM Journal of Applied Mathematics, 58, (1998), 211-226.

[8] Szentruti, Zs.: Periodic solutions in non-symmetric May-Leonard systems, Publications of the University Miskolc, Series D. Natural Sciences. Mathematics, 40, (1999), 89-103. 